\title{
Provisioning Algorithms for WDM Optical Networks
}

\author{
Murat Alanyali, Member, IEEE, and Ender Ayanoglu, Fellow, IEEE
}

\begin{abstract}
This paper concerns connection provisioning for optical networks employing wavelength division multiplexing. A heuristic algorithm is developed and numerically studied for routing and wavelength assignment of a set of static connection requests. The algorithm runs much faster than the optimum solution of this problem. An adaptation of the algorithm is proposed to design restorable networks which can handle a specified set of failures. The proposed algorithm is based on taking all failures into consideration simultaneously, and performs better than developing independent designs for each failure.
\end{abstract}

\section{INTRODUCTION}

$\mathbf{T}$ RANSPORT networks are WAN's that provide connectivity for aggregated traffic streams. Modern transport networks increasingly employ wavelength division multiplexing (WDM) technology to utilize the vast transmission bandwidth of fiber. WDM is based on transmission of data over separate wavelength channels on each fiber. At the present time, WDM is mainly employed as a point-to-point transmission technology. In such networks, optical signals on each wavelength are converted to electrical signals at each network node. On the other hand, WDM optical networking technology, which has been developed within the last decade and is becoming commercially available [1]-[3], employs wavelengths on an end-to-end basis, without electrical conversion in the network.

Provisioning of a transport network refers to assigning network resources to a static traffic demand. Efficient provisioning is essential in minimizing the investment made on the network required to accommodate a given demand. In the context of WDM optical networks, provisioning means routing and wavelength selection for a set of end-to-end wavelength allocation demands, given a demand distribution and a network topology. Naturally, provisioning of WDM networks has been subject to considerable interest. This interest concentrates on roughly two categories of settings: the case of limited deployed fiber, where provisioning seeks to minimize the number of required wavelengths [4]-[6], and the case of limited number of wavelengths per fiber, where provisioning

Manuscript received February 2, 1998; revised March 18, 1999; approved by IEEE/ACM Transactions on NeTworking Editor K. N. Sivarajan. This work was supported in part by Defence Advanced Research Projects Agency under the Multiwavelength Optical Networking Consortium of Lucent Technologies, AT\&T, Bellcore, Bell Atlantic, Bell South, and SBC, with the participation of the Naval Research Laboratory and the National Security Agency.

M. Alanyali is with the Department of Electrical and Electronics Engineering, Bilkent University, Bilkent, Ankara TR-06533, Turkey (e-mail: alanyali@ee.bilkent.edu.tr).

E. Ayanoglu is with Cisco Systems, San Jose, CA 95134 USA (e-mail: ender@ieee.org).

Publisher Item Identifier S 1063-6692(99)08251-5. seeks to minimize the amount of required fiber [7], [8] or to maximize accommodated traffic [9].

In this paper, we focus on heuristic methods for provisioning a static set of connections on a given WDM optical network topology. It is assumed that each connection requires a dedicated wavelength on each link of its path. We consider the setting where there is a fixed set of wavelengths available on each fiber and, therefore, the connections are established at the expense of possibly multiple fibers on network links. Each fiber has a cost which reflects the fiber material, optical amplifiers, and the optical termination equipment at both ends of the link. The objective of provisioning is taken as the minimization of the total network cost.

Since transport networks are chartered to carry high volumes of traffic, network failures may have severe consequences. This imposes fault-tolerance as an essential feature for transport networks. Fault-tolerance refers to the ability of the network to reconfigure and reestablish communication upon failure, and is widely known as restoration. Restoration entails rerouting connections around failed components under a targeted time-to-restore. A network with restoration capability requires redundant capacity to be used in the case of failures, and a primary concern in designing such networks is to provide robustness with minimal redundancy. In principle, design methods devised for conventional, single-wavelength restorable networks [10]-[12] can be employed in WDM optical networks as well. Such designs prescribe switching all wavelengths in a fiber together in the case of failure. WDM optical networking, however, provides the capability to switch individual wavelengths, thereby offers a richer set of design methods [13]-[15].

The paper is comprised of two parts. The first part concerns provisioning in networks that do not account for restoration. Such a network is called a primary network, and the objective in primary-network design is to minimize the cost associated with the working fibers. This problem can be formulated as an integer linear program (ILP) in a straightforward manner; however, the computational complexity of the ILP is prohibitive for a network whose size is not trivial. Therefore, efficient heuristic solution methods are needed. We present such a heuristic algorithm that produces good solutions several orders of magnitude faster than general-purpose ILP packages. The second part of the paper involves an adaptation of this heuristic to provisioning in restorable networks. We consider precomputed restoration schemes where the reaction of the network to certain failures is computed in advance, so that upon each failure, a certain reconfiguration is adopted. The heuristic algorithm entails determining all reconfigurations simultaneously, in a coordinated manner. This results in better 
designs compared to methods in which the configurations are developed independently for different failures.

The discussion in the paper is limited to wavelengthselective WDM optical networks: it is assumed that each connection occupies the same wavelength on its entire path. Nevertheless, the algorithm easily extends to networks in which an arbitrary subset of nodes have wavelength conversion capability.

The outline of the paper is as follows. The primary-network design problem is studied in Section II. Essential definitions and a mathematical formulation of the problem are provided. Section II-A concentrates on a related but simpler problem, whose solution leads to a heuristic algorithm for the original problem. This algorithm is the subject of Section II-B, and some variations of the algorithm are discussed in Section IIC. Section III concentrates on the restorable-network design problem. The problem is formulated, and an adaptation of the heuristic algorithm is proposed as an efficient heuristic solution method. The performances of the proposed heuristics are observed numerically in Section IV. Finally, concluding remarks are presented in Section $\mathrm{V}$.

\section{PRIMARY-NETWORK DESIGN}

We start the formulation of the primary-network design problem by providing some essential definitions which are used throughout the paper. In this paper a network is represented by an undirected, weighted graph $G=(N, E, D)$. Here, $N$ denotes a set of nodes, $E$ denotes a set of links, and $D=(d(e): e \in E)$ denotes a set of positive link weights. In the envisioned applications, the weight of a link represents the cost per fiber deployed on the link. The set of wavelengths that are available on each fiber is identified by $W$. Given a link $e$, a path $p$, and a wavelength $w$, the pair $(e, w)$ is called a lighthop, and the pair $(p, w)$ is called a lightpath. Let $U=N \times N$ denote the set of node pairs in the network. The demand for the network is an integer vector $\delta=(\delta(u): u \in U)$, where $\delta(u)$ denotes the number of connections to be established between the pair of nodes in $u{ }^{1}$ It is assumed that in the primary network, each such connection can only be routed on a path from a prespecified set of admissible paths, which is identified by $P(u)$. If $p \in P(u)$ and $w \in W$, then we call the pair $(p, w)$ an admissible lightpath for the node pair $u$.

An assignment is defined as a real vector $a=$ $(a(p, w): p \in P(u), u \in U, w \in W)$ such that $a \geq 0$ and $\sum_{p \in P(u)} \sum_{w \in W} a(p, w)=\delta(u)$ for each $u \in U$. Under assignment $a$ the load at lighthop $(e, w), x(e, w)$, is expressed as

$$
x(e, w)=\sum_{u \in U} \sum_{p \in P(u): e \in p} a(p, w) .
$$

If the vector $a$ is an integer assignment then $a(p, w)$ represents the number of connections that are assigned to the lightpath $(p, w)$. Each such connection occupies the wavelength $w$ on each link of the path $p$. Therefore, if the integer

\footnotetext{
${ }^{1}$ Connections are taken to be bidirectional mainly for simplicity of presentation. Subsequent discussion and algorithms extend in a straightforward manner to the case of unidirectional connections.
}

TABLE I

The Size of the ILP Formulation of the Primary-Network Design Problem for Several Mesh-Like Networks

\begin{tabular}{l|c|c|c}
\hline \multirow{2}{*}{} & \multicolumn{3}{|c}{ number of nodes } \\
\cline { 2 - 4 } & 9 & 32 & 88 \\
\hline number of variables & 698 & 8,978 & 68,954 \\
number of constraints & 340 & 1,888 & 11,884 \\
\hline
\end{tabular}

In each case, the number of wavelengths is eight and the number of admissible paths per node pair is two.

assignment $a$ is adopted, then the number of fibers required at link $e$ is given by $\max _{w \in W} x(e, w)$. The cost corresponding to $a, J(a)$, is then defined as

$$
J(a)=\sum_{e \in E} d(e) \max _{w \in W} x(e, w) .
$$

We can now formally state the design problem considered in this section. Given a network $G$, wavelength set $W$, demand $\delta$, and admissible paths $(P(u): u \in U)$, the primary-network design problem $P$ consists of assigning an admissible lightpath to each connection so as to minimize the resulting cost. Namely,

$$
P: \min \{J(a): a \text { is an integer assignment }\} .
$$

The problem $P$ can easily be formulated as an integer linear problem as follows:

$$
\min \sum_{e \in E} d(e) \kappa(e) \text { such that } x(e, w) \leq \kappa(e) \text { for each }
$$

$e \in E, w \in W$, and $a$ is an integer assignment.

Here $(\kappa(e): e \in E)$ and $(a(p, w): p \in P(u), u \in U, w \in$ $W)$ are variables, whereas $(x(e, w): e \in E, w \in W)$ is determined by equality (1). Typical numbers of variables and constraints in the ILP formulation are listed in Table I for some mesh-like topologies of various sizes. General-purpose codes for solving ILP's typically employ branch and bound techniques with linear programming relaxation. As indicated in Section IV, such solution methods generally entail a very high computational complexity, even for moderate-size networks, particularly if there is a large number of connections to be established. In view of this, we focus on heuristic solution methods, and in the rest of this section introduce an efficient heuristic algorithm.

\section{A. A Motivating Optimization Problem}

In this section, we briefly digress from primary-network design, and introduce a collection of auxiliary optimization problems which are parametrized by a positive scalar $\alpha$. These problems are related to the primary-network design problem $P$ in that, as $\alpha$ grows larger, their cost functions converge to the function $J$. Proposition 2.1 provides a characterization of solutions to each auxiliary problem, which in turn inspires a heuristic solution method for problem $P$.

We start with some definitions. Given a positive number $\alpha$, let $J^{\alpha}$ denote a cost function, such that for each assignment $a$

$$
J^{\alpha}(a)=\alpha^{-1} \sum_{e \in E} d(e) \log \sum_{w \in W} \exp (\alpha x(e, w)) .
$$


Note that

$$
\begin{aligned}
J^{\alpha}(a)= & \sum_{e \in E} d(e) \max _{w \in W} x(e, w)+\alpha^{-1} \sum_{e \in E} d(e) \\
& \log \sum_{w \in W} \exp \left(\alpha\left(x(e, w)-\max _{w^{\prime} \in W} x\left(e, w^{\prime}\right)\right)\right)
\end{aligned}
$$

and therefore

$$
J(a) \leq J^{\alpha}(a) \leq J(a)+\alpha^{-1} \sum_{e \in E} d(e) \log |W| .
$$

In particular, $\lim _{\alpha \rightarrow \infty} J^{\alpha}(a)=J(a)$ uniformly for all assignments $a$. Finally, let the optimization problem $P^{\alpha}$ be defined as follows:

$$
P^{\alpha}: \min \left\{J^{\alpha}(a): a \text { is a real assignment }\right\} .
$$

Notice that in contrast to the problem $P$, solutions of $P^{\alpha}$ are not constrained to be integer-valued assignments.

We next study possible solutions of $P^{\alpha}$. Toward this end, note the differentiability of $J^{\alpha}$, and let $M^{\alpha}(p, w)$ denote its partial derivative with respect to $a(p, w)$. Namely, given an assignment $a$ with corresponding load $x$

$$
M^{\alpha}(p, w)=\frac{\partial J^{\alpha}(a)}{\partial a(p, w)}=\sum_{e \in p} d(e) \frac{\exp (\alpha x(e, w))}{\sum_{w^{\prime} \in W} \exp \left(\alpha x\left(e, w^{\prime}\right)\right)} .
$$

If $(p, w)$ and $\left(p^{\prime}, w^{\prime}\right)$ are admissible lightpaths for a node pair $u$ such that $a(p, w)>0$ and $M^{\alpha}(p, w)>M^{\alpha}\left(p^{\prime}, w^{\prime}\right)$, then one can find a small, positive number $\sigma$ such that decreasing $a(p, w)$ by $\sigma$ and increasing $a\left(p^{\prime}, w^{\prime}\right)$ by $\sigma$ results in another assignment whose cost is strictly less than $J^{\alpha}(a)$. Thus, if an assignment $a$ is optimal for problem $P^{\alpha}$, then for each node pair $u$ and each admissible lightpath $(p, w)$ of $u$,

$$
\begin{aligned}
a(p, w) & =0 \quad \text { whenever } \\
M^{\alpha}(p, w) & >\min \left\{M^{\alpha}\left(p^{\prime}, w^{\prime}\right):\left(p^{\prime}, w^{\prime}\right) \text { admissible for } u\right\} .
\end{aligned}
$$

The following proposition establishes that this condition is also sufficient for optimality in $P^{\alpha}$. The proof of the proposition is given in the Appendix.

Proposition 2.1: There exists a solution to the problem $P^{\alpha}$. An assignment $a$ solves $P^{\alpha}$ if and only if condition (2) holds for each node pair $u$ and each admissible lightpath $(p, w)$ of $u$.

Proposition 2.1 does not provide a constructive characterization for the solutions of problem $P^{\alpha}$. However, such solutions can be well approximated by employing steepest descent methods. In the context of optimal routing, such methods are referred to as flow deviation algorithms. In the present context, flow deviation entails identifying a lightpath that has the smallest partial derivative $M^{\alpha}$, and obtaining a better assignment by increasing the assignment on that lightpath by an appropriate value. In particular, one can employ a sequence of flow deviations to arrive at a real assignment that is nearoptimal for problem $P^{\alpha}$ (for a sketch of proof, consult [16, Problem 5.31] together with Proposition 2.1). In the following section, we use an adaptation of this idea to develop a heuristic, iterative procedure to obtain good integer assignments for the original problem $P$.

\section{B. The Heuristic Design Algorithm}

In this section, we return to the primary-network design problem, and describe a heuristic solution method. The method relies on a metric defined on the lightpaths of the network. Namely, given an assignment $a$ with the corresponding load $x$, the lightpath-metric $M(p, w)$ of a lightpath $(p, w)$ is defined as

$$
M(p, w)=\sum_{e \in p} m(e, w)
$$

where

$$
m(e, w)= \begin{cases}d(e) / n_{e}(a) & \text { if } x(e, w)=\max _{w^{\prime} \in W} x\left(e, w^{\prime}\right) \\ 0 & \text { else }\end{cases}
$$

and $n_{e}(a)=\left|\left\{\tilde{w} \in W: x(e, \tilde{w})=\max _{w^{\prime}} x\left(e, w^{\prime}\right)\right\}\right|$ is the number of wavelengths that have the maximum load on link e. Note that

$$
M(p, w)=\lim _{\alpha \rightarrow \infty} M^{\alpha}(p, w) .
$$

To facilitate the description of the algorithm, it is convenient to identify each connection in the network separately. Toward this end, for each node pair $u$, let $\Delta(u)$ denote the set of connections to be established between $u$. In particular $|\Delta(u)|=\delta(u)$. A configuration is defined as a mapping from the connections into the associated admissible lightpaths, and identifies a path and wavelength selection for each connection. Given a configuration, let $p(c)$ and $w(c)$ denote, respectively, the path and wavelength assigned to connection $c$. Also let the cumulative metric of the network denote the sum $\sum_{u \in U} \sum_{c \in \Delta(u)} M(p(c), w(c))$.

A configuration uniquely identifies an integer assignment $a$. We thus define the cost of a configuration as the cost $J(a)$ of the corresponding assignment. Let $x$ denote the load corresponding to $a$. A straightforward calculation yields that

$$
\begin{aligned}
\sum_{u \in U} \sum_{c \in \Delta(u)} M(p(c), w(c)) & =\sum_{e \in E} \sum_{w \in W} x(e, w) m(e, w) \\
& =\sum_{e \in E} d(e) \max _{w \in W} x(e, w) \\
& =J(a)
\end{aligned}
$$

hence the cost of a configuration is given by the cumulative metric of the network. This observation, along with the flow deviation approach of Section II-A, inspires a heuristic algorithm which we describe next.

The algorithm starts with an arbitrary initial configuration, and iteratively computes new configurations until a certain stopping criterion is fulfilled. An iteration involves one decision by each node pair. Namely, each node pair $u$ first determines a connection $c \in \Delta(u)$ that maximizes the lightpath-metric $M(p(c), w(c))$ in the current configuration. A new configuration is then obtained by setting $(p(c), w(c))=$ $(p, w)$, where $(p, w)$ is an admissible lightpath for $u$ such that the lightpath-metric $M(p(c), w(c))$ is minimized under the resulting configuration. The flowchart of an iteration is given in Fig. 1. The output of the algorithm is a configuration that achieves the smallest cost in the course of the iterations. 


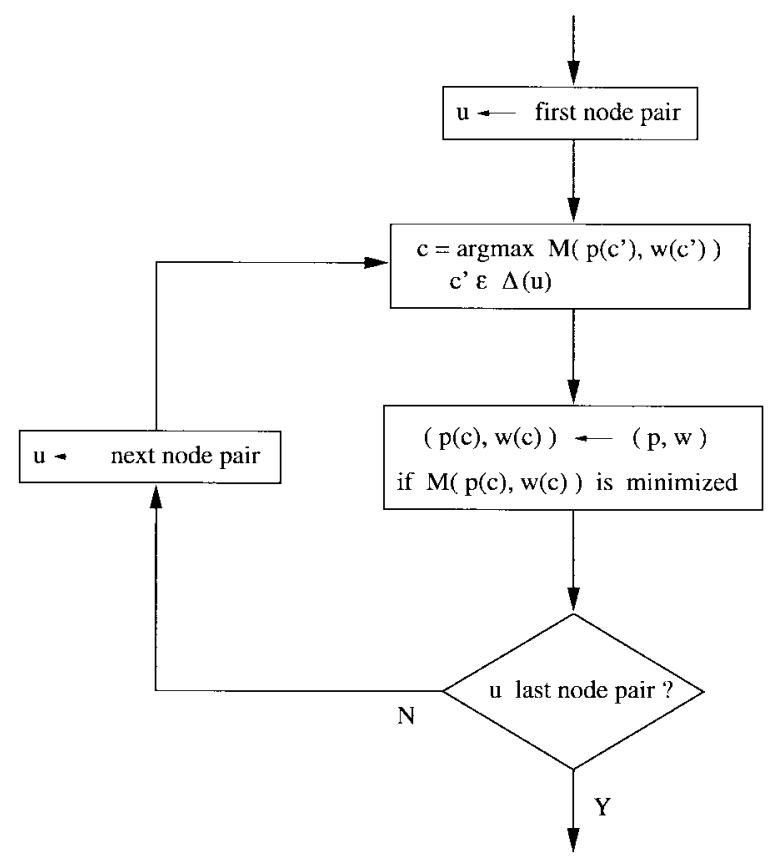

Fig. 1. Flowchart of an iteration of the primary-network design algorithm.

Example: We now illustrate one iteration of the algorithm on a sample network topology depicted in Fig. 2. Consider the case in which there are four wavelengths (i.e., $|W|=4$ ), only the first two shortest paths are admissible for each node pair (hence, $k=2$ ) and $d(e)=1$ for each link $e$. The demand, the initial configuration of the network, and the resulting lighthop occupancies are given in Fig. 2(a). Note that this initial configuration has cost 6 . Suppose that within an iteration node pairs are considered in the order $((1,2),(1,4),(2,3),(2,4),(3,4))$. The algorithm takes the following actions in the indicated order.

1) Node pair $(1,2)$ is considered. Since $c 1$ is the only connection, it is marked for rerouting. Each of the eight alternative lightpaths $(p, w)$ to reroute $c 1$ is evaluated by computing the metric $M(p, w)$ in a network configuration in which $c 1$ is routed on $(p, w)$ and all other connections maintain their current lightpath assignments. For example the metric of lightpath $((1 \rightarrow 3 \rightarrow 2), 4)$ is $1+1 / 3=4 / 3$, since if $c 1$ is routed on this lightpath, then wavelength occupancies of links $(1 \rightarrow 3)$ and $(3 \rightarrow 2)$ would be $(0,0,0,1)$ and $(1,1,0,1)$, respectively. The eight metrics are listed in Table II. The smallest metric is observed when $(p, w)=((1 \rightarrow 2), 3)$, thus connection $c 1$ is rerouted on this lightpath. The resulting network configuration is given in Fig. 2(b). Note that the network cost is decreased by one after this step.

2) Node pair $(1,4)$ is considered. In the current configuration, both connections $c 1$ and $c 2$ are assigned to lightpaths with identical metrics (that being $1 / 3+$ $1 / 2$ ), thus without loss of generality $c 1$ is marked for rerouting. If connection $c 1$ is rerouted on the lightpath $(p, w)=((1 \rightarrow 2 \rightarrow 4), 4)$, then $(p, w)$ has metric $M(p, w)=1 / 3$ in the resulting configuration. Straightforward calculation yields that $1 / 3$ is the minimum of
TABLE II

Metrics of Alternative LightPATHS for Connection $c 1$ of Node Pair $(1,2)$

\begin{tabular}{l|c}
\hline Lightpath $(p, w)$ & $M(p, w)$ \\
\hline$((1 \rightarrow 2), 1)$ & 1 \\
$((1 \rightarrow 2), 2)$ & 1 \\
$((1 \rightarrow 2), 3)$ & $1 / 3$ \\
$((1 \rightarrow 2), 4)$ & $1 / 3$ \\
$((1 \rightarrow 3 \rightarrow 2), 1)$ & 2 \\
$((1 \rightarrow 3 \rightarrow 2), 2)$ & 2 \\
$((1 \rightarrow 3 \rightarrow 2), 3)$ & $4 / 3$ \\
$((1 \rightarrow 3 \rightarrow 2), 4)$ & $4 / 3$ \\
\hline
\end{tabular}

metrics similarly obtained for all admissible lightpaths, therefore $c 1$ is rerouted on $(p, w)$. The resulting configuration is given in Fig. 2(c). Note that this step does not further decrease the cost.

3) Node pair $(2,3)$ is considered, and connection $c 1$ is marked for rerouting. No alternative lightpath yields a smaller metric than the current lightpath; thus, the current configuration is not altered.

4) Node pair $(2,4)$ is considered. In the current configuration, lightpath of connection $c 2$ has the maximum metric (which is 1 ), therefore $c 2$ is marked. Consideration of alternative lightpaths leads to rerouting $c 2$ on lightpath $(p, w)=((2 \rightarrow 3 \rightarrow 4), 3)$, which has metric $M(p, w)=1 / 3+1 / 2$ in the resulting configuration. That configuration is given in Fig. 2(d). This step results in decreasing the cost by one.

5) Node pair $(3,4)$ is considered. The current lightpath of connection $c 1$ attains the minimal metric among all alternatives, in turn the configuration is not altered.

During the execution of the algorithm, node pairs greedily attempt to decrease the maximum lightpath metric observed by their connections. The heuristic aim of this effort is to obtain a configuration with minimal cumulative metric, and thereby, in view of identity (3), minimize the network cost. Note that while this effort reduces the metric observed by the most recently rerouted connection, it may increase the metrics observed by other connections, in turn the network cost is not necessarily monotone with respect to iterations. ${ }^{2}$ A typical variation of the network cost with iteration number is plotted in Fig. 3.

The numerical study of Section IV suggests that the performance of the algorithm depends rather weakly on the initial network configuration and on the order in which node pairs are considered within an iteration. Heuristic methods for selecting these parameters are briefly discussed in Section IV.

The computational complexity per iteration of the algorithm is linear in the total number of connections and in the total number of admissible lightpaths. As reported in Section IV, the algorithm typically produces good configurations in a small number of iterations; thus, it provides a fast suboptimal alternative to the integer programming approach.

\footnotetext{
${ }^{2}$ One can easily detect and avoid such reroutings; however, enforcing a monotone convergence in this manner is likely to limit the performance of the algorithm due to high-cost local minima.
} 


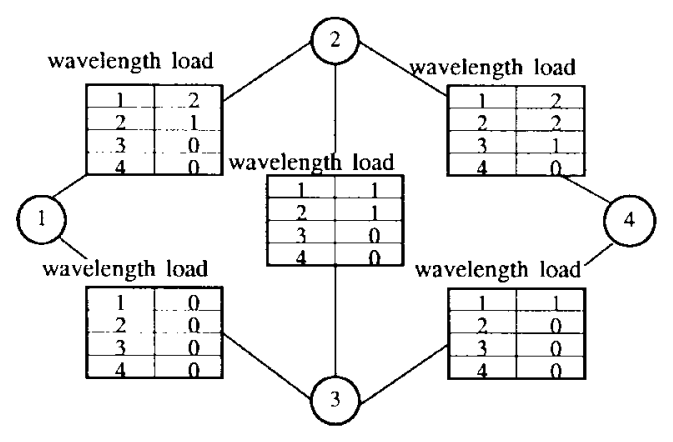

\begin{tabular}{l|c|c|l|c} 
s-d pair $(u)$ & demand $\delta(u)$ & demand set $\Lambda(u)$ & configuration $(p(c), w(c))$ & metric $M(p(c), w(c))$ \\
\hline$(1,2)$ & 1 & $c 1$ & $((1->2), 1)$ & 1 \\
\hline$(1,4)$ & 2 & $c 1$ & $((1->2->4), 1)$ & $1+1 / 2$ \\
& & $c 2$ & $((1->2->4), 2)$ & $1 / 2$ \\
\hline$(2,3)$ & 2 & $c 1$ & $((2->3), 1)$ & $1 / 2$ \\
& & $c 2$ & $((2->3), 2)$ & $1 / 2$ \\
\hline$(2,4)$ & 3 & $c 1$ & $((2->4), 1)$ & $1 / 2$ \\
& & $c 2$ & $((2->4), 2)$ & $1 / 2$ \\
& & $c 3$ & $((2->4), 3)$ & 0 \\
\hline$(3,4)$ & 1 & $c 1$ & $((3->4), 1)$ &
\end{tabular}

(a)

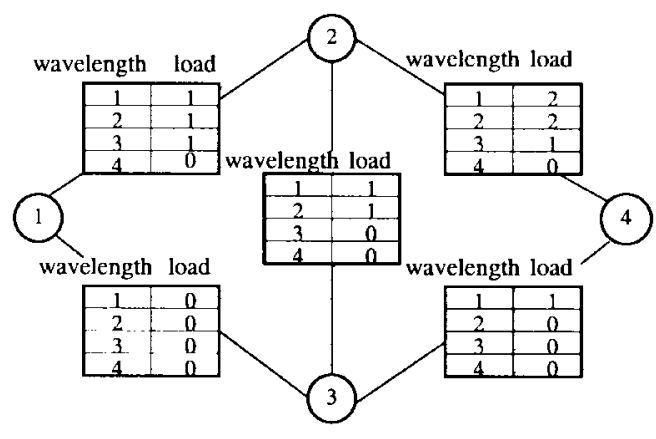

\begin{tabular}{l|c|c|l|c}
$\mathrm{s}-\mathrm{d}$ pair $(\mathrm{u})$ & demand $\delta(\mathrm{u})$ & demand set $\Delta(\mathrm{u})$ & configuration $(\mathrm{p}(\mathrm{c}), \mathrm{w}(\mathrm{c}))$ & mctric $\mathrm{M}(\mathrm{p}(\mathrm{c}), \mathrm{w}(\mathrm{c}))$ \\
\hline$(1,2)$ & 1 & $\mathrm{c} 1$ & $((1->2), 3)$ & $1 / 3$ \\
\hline$(1,4)$ & 2 & $\mathrm{cl}$ & $((1->2->4), 1)$ & $1 / 3+1 / 2$ \\
& & $\mathrm{c} 2$ & $((1->2->4), 2)$ & $1 / 3+1 / 2$ \\
\hline$(2,3)$ & 2 & $\mathrm{c} 1$ & $((2->3), 1)$ & $1 / 2$ \\
& & $\mathrm{c} 2$ & $((2->3), 2)$ & $1 / 2$ \\
\hline$(2,4)$ & 3 & $c 1$ & $((2->4), 1)$ & $1 / 2$ \\
& & $c 2$ & $((2->4), 2)$ & $1 / 2$ \\
& & $\mathrm{c3}$ & $((2->4), 3)$ & 0 \\
\hline$(3,4)$ & 1 & $\mathrm{c1}$ & $((3->4), 1)$ & 1
\end{tabular}

(b)

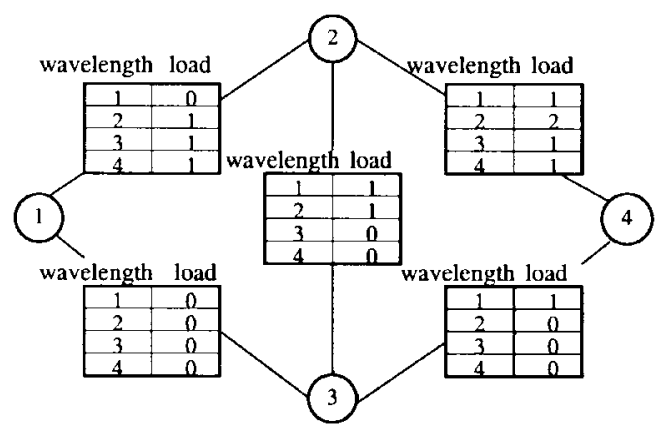

\begin{tabular}{c|c|c|l|c} 
s-d pair $(\mathrm{u})$ & demand $\delta(\mathrm{u})$ & demand set $\Delta(\mathrm{u})$ & configuration $(\mathrm{p}(\mathrm{c}), \mathrm{w}(\mathrm{c}))$ & metric $\mathrm{M}(\mathrm{p}(\mathrm{c}), \mathrm{w}(\mathrm{c}))$ \\
\hline$(1,2)$ & 1 & $\mathrm{cl}$ & $((1->2), 3)$ & $1 / 3$ \\
\hline$(1,4)$ & 2 & $\mathrm{cl}$ & $((1->2->4), 4)$ \\
& & $\mathrm{c} 2$ & $((1->2->4), 2)$ & $1 / 3$ \\
& 2 & $\mathrm{cl}$ & $((2->3), 1)$ & $1 / 3+1$ \\
\hline$(2,3)$ & & $\mathrm{c} 2$ & $((2->3), 2)$ & $1 / 2$ \\
& 3 & $\mathrm{cl}$ & $((2->4), 1)$ & $1 / 2$ \\
\hline$(2,4)$ & & $\mathrm{c} 2$ & $((2->4), 2)$ & 1 \\
& & $\mathrm{c3}$ & $((2->4), 3)$ & 0 \\
\hline$(3,4)$ & 1 & $\mathrm{cl}$ & $((3->4), 1)$ & 1
\end{tabular}

(c)

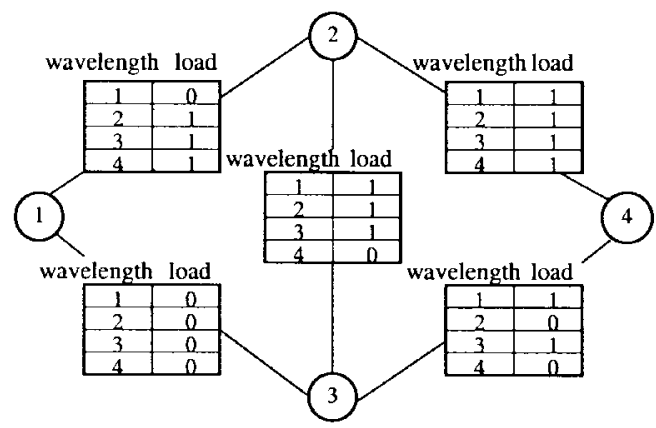

\begin{tabular}{c|c|c|l|c} 
s-d pair (u) & demand $\delta(\mathrm{u})$ & demand set $\Delta(\mathrm{u})$ & configuration $(\mathrm{p}(\mathrm{c}), \mathrm{w}(\mathrm{c}))$ & metric $\mathrm{M}(\mathrm{p}(\mathrm{c}), \mathrm{w}(\mathrm{c}))$ \\
\hline$(1,2)$ & 1 & $\mathrm{c} 1$ & $((1->2), 3)$ & $1 / 3$ \\
\hline$(1,4)$ & 2 & $\mathrm{c} 1$ & $((1->2->4), 4)$ & $1 / 3+1 / 4$ \\
& & $c 2$ & $((1->2->4), 2)$ & $1 / 3+1 / 4$ \\
\hline$(2,3)$ & 2 & $\mathrm{cl}$ & $((2->3), 1)$ & $1 / 3$ \\
& & $c 2$ & $((2->3), 2)$ & $1 / 3$ \\
\hline$(2,4)$ & 3 & $c 1$ & $((2->4), 1)$ & $1 / 4$ \\
& & $c 2$ & $((2->3->4), 3))$ & $1 / 3+1 / 2$ \\
& & $c 3$ & $((2->4), 3)$ & $1 / 4$ \\
\hline$(3,4)$ & 1 & $c 1$ & $((3->4), 1)$ & $1 / 2$
\end{tabular}

(d)

Fig. 2. The sample network design problem to illustrate phases of one iteration of the algorithm.

\section{Alternative Metrics}

One can think of a class of heuristic algorithms, each of which is obtained by adopting a different lightpath-metric in the algorithm described in Section II-B. In this section, we briefly discuss five such variations as listed in Table III. Dynamic versions of these algorithms have been considered in certain network management settings, it is thus of interest to see their performances in the primary-network design problem. We highlight the following observations.

i) Since $\max _{w^{\prime} \in W} x\left(e, w^{\prime}\right)$ denotes the capacity requirement of link $e$, the $m N S$ algorithm of Table III tends to reroute connections on lightpaths whose lighthops 
TABLE III

Variations of the Heuristic Primary-Network Design Algorithm

\begin{tabular}{l|l}
\hline Algorithm & Lightpath-metric of $(p, w)$ \\
\hline Min normalized sum $(m N S)$ & $M_{m N S}(p, w)=\sum_{e \in p} x(e, w) / \max _{w^{\prime} \in W} x\left(e, w^{\prime}\right)$ \\
Min normalized product $(m N P)$ & $M_{m N P}(p, w)=\sum_{e \in p} \log \left(x(e, w) / \sum_{w^{\prime} \in W} x\left(e, w^{\prime}\right)\right)$ \\
Max residual capacity $(M R C)$ & $M_{M R C}(p, w)=\sum_{e \in p}\left(x(e, w)-\max _{w^{\prime} \in W} x\left(e, w^{\prime}\right)\right)$ \\
Max normalized res. cap. $(M N R C)$ & $M_{M N R C}(p, w)=\sum_{e \in p}\left(\left(x(e, w) / \max _{w^{\prime} \in W} x\left(e, w^{\prime}\right)\right)-1\right)$ \\
Max-min residual capacity $(M m R C)$ & $M_{M m R C}(p, w)=-\min _{e \in p}\left(\max _{w^{\prime} \in W} x\left(e, w^{\prime}\right)-x(e, w)\right)$ \\
\hline
\end{tabular}

$\operatorname{Cost}$

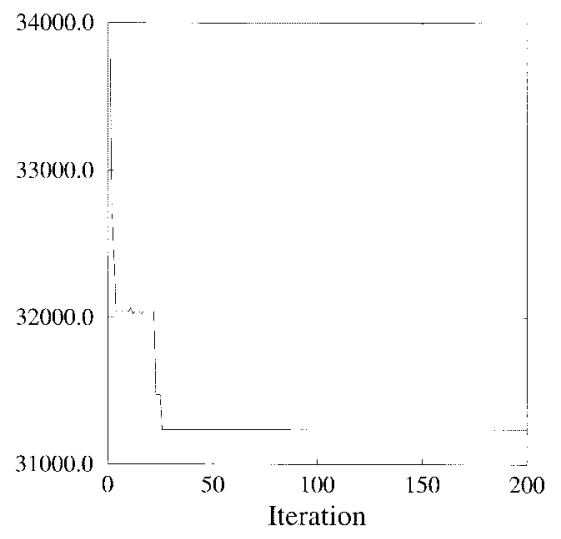

Fig. 3. Typical variation of the cost with respect to the iterations for the heuristic primary-network design algorithm. The initial configuration is also constructed via the same heuristic, therefore the iterations start with an already small cost.

are lightly loaded, and it is likely to result in a high wavelength utilization on each link of the network. A straightforward calculation yields that the cumulative $(m N S)$ metric of a configuration is given by

$$
\begin{aligned}
& \sum_{u \in U} \sum_{c \in \Delta(u)} M_{m N S}(p(c), w(c)) \\
& \quad=\sum_{e \in E} \max _{w^{\prime} \in W} x\left(e, w^{\prime}\right) \sum_{w \in W}\left(x(e, w) / \max _{w^{\prime} \in W} x\left(e, w^{\prime}\right)\right)^{2} .
\end{aligned}
$$

Hence, roughly speaking, minimizing the cumulative metric entails equalizing the loads on the lighthops of each link, as well as minimizing the total capacity requirement.

ii) While the $m N P$ algorithm favors lightpaths with lightly-loaded lighthops, it may also favor long lightpaths. Define $H(e)=\sum_{w \in W}\left(x(e, w) / \sum_{w^{\prime} \in W}\right.$ $\left.x\left(e, w^{\prime}\right)\right) \log \left(x(e, w) / \sum_{w^{\prime} \in W} x\left(e, w^{\prime}\right)\right)$ for each link $e$, so that $\sum_{u \in U} \sum_{c \in \Delta(u)} M_{m N P}(p(c), w(c))=$ $\sum_{e \in E}\left(\sum_{w^{\prime} \in W} x\left(e, w^{\prime}\right)\right) H(e)$. Note that minimizing the cumulative metric of the network entails a high wavelength utilization, so that $H(e)$ is small, but typically a high capacity requirement, since $H(e)$ is nonpositive.

iii) The $M R C$ algorithm also may favor long lightpaths of lightly loaded lighthops. The cumulative metric of this algorithm is given by $\sum_{e \in E} \sum_{w \in W}$ $x(e, w)\left(x(e, w)-\max _{w^{\prime} \in W} x\left(e, w^{\prime}\right)\right)$, hence its minimization may imply a high capacity requirement since $x(e, w)-\max _{w^{\prime} \in W} x\left(e, w^{\prime}\right)$ is nonpositive.
Note that these observations apply to the $M N R C$ algorithm as well.

iv) The $M m R C$ algorithm reassigns connections so as to avoid bottleneck lighthops, and it is shown to be effective in dynamic settings [17].

On the premise that iterations tend to cluster around configurations that minimize the cumulative metric of the network, one may expect the algorithm $m N S$ to perform well, whereas algorithms $m N P, M R C$, and $M N R C$ may be expected to yield somewhat high network cost, despite good wavelength utilization. This intuition is supported by a numerical evaluation of the algorithms which is provided in Section IV.

\section{Restorable-Network Design}

This section concerns the provisioning of networks that have restoration capability. The aim of the provisioning methods considered here is to minimize the network cost while providing sufficient capacity to guarantee network robustness against a specified set of failures. We consider the case when a primary-network configuration is fixed in advance, and assume that upon each failure connections are sustained by adopting a predetermined reconfiguration. Two heuristic methods to obtain such reconfigurations are introduced next. The performances of these methods are numerically evaluated in the following section.

We start with a number of definitions which are helpful in the formulation of the design problem. Suppose that a primary-network configuration is given, so that under normal operation conditions (i.e., no failures), the lightpath assignment of each connection is known. A failure scenario is defined as a collection of failed network components. Here a network component may denote a link or a node. The set of connections that are allowed to be reconfigured upon a failure scenario $f$ is referred to as the impact set of the failure scenario, and is denoted by $I_{f}$. This set is a design parameter, however it necessarily includes the connections that utilize at least one of the failed components in the primary-network configuration. Larger impact sets yield more efficient designs at the expense of larger restoration time and complexity. Upon failure scenario $f$, each connection $c$ in the impact set $I_{f}$ needs to be reassigned a lightpath from the set of restoration paths for $c$, denoted by $P_{f}(c)$. Naturally, the paths in the set $P_{f}(c)$ may not utilize any of the components in the failure scenario $f$. This set also takes on different forms for linkbased and path-based restoration schemes, as explained in Section IV. Finally, a reconfiguration for the failure scenario $f$ refers to a lightpath assignment in which the connections in the impact set $I_{f}$ are assigned restoration paths, whereas 
the remaining connections retain the same lightpaths as in the primary-network configuration.

Each reconfiguration identifies an integer vector on the lightpaths of the network. We now provide a description of this vector, which will be useful in the mathematical formulation of the problem. Consider a reconfiguration for failure scenario $f$, and let $a_{f}(p, w)$ denote the number of connections assigned to lightpath $(p, w)$. Also let $b_{f}(p, w)$ be the number of connections that are assigned the lightpath $(p, w)$ in the primary-network configuration, but do not belong to the impact set $I_{f}$. Note that each such connection needs to be assigned the same lightpath in the reconfiguration, therefore $a_{f}(p, w) \geq b_{f}(p, w)$. For each node pair $u$, let $P_{f}(u)=$ $\cup_{c \in \Delta(u) \cap I_{f}} P_{f}(c)$ denote the restoration paths associated with $u$. The total number of connections on these paths is no less than the number of reconfigured connections, therefore $\sum_{p \in P_{f}(u)} \sum_{w \in W} a_{f}(p, w) \geq\left|\Delta(u) \cap I_{f}\right|$ for $u \in U$. Finally, the reconfiguration should satisfy the demand, and thus $\sum_{p \in P(u) \cup P_{f}(u)} \sum_{w \in W} a_{f}(p, w)=\delta(u)$. An integer vector which satisfies the above conditions is called a reassignment for failure scenario $f$. Note also that a reconfiguration can easily be constructed from such a vector.

Design of a restorable network entails determining reassignments for a collection of envisioned failure scenarios. Let $F$ be such a collection. A set $A_{F}=\left(a_{f}: f \in F\right)$ of one reassignment for each failure scenario in $F$ is called a restoration program for $F$. A restoration program identifies a reconfiguration to be adopted upon each failure scenario. Let $x$ denote the load corresponding to the primary-network configuration, and for each $f \in F$, let $x_{f}$ denote the load under reassignment $a_{f}$. For each lighthop $(e, w)$, define $x^{*}(e, w)=$ $\max \left\{x(e, w), \max _{f \in F} x_{f}(e, w)\right\}$. Note that if restoration program $A_{F}$ is adopted, then the capacity requirement at a link $e$ is $\max _{w \in W} x^{*}(e, w)$, so that all connections are guaranteed connectivity under at most one failure scenario from $F$. The cost of the restoration program $A_{F}$ is now defined as

$$
J^{*}\left(A_{F}\right)=\sum_{e \in E} d(e) \max _{w \in W} x^{*}(e, w) .
$$

Given a primary-network configuration, a collection of failure scenarios $F$, impact sets $\left(I_{f}: f \in F\right)$, and restoration paths $\left(P_{f}(c): f \in F, c \in I_{f}\right)$, we define the restorablenetwork design problem $R$ as follows:

$$
R: \min \left\{J^{*}\left(A_{F}\right): A_{F} \text { is a restoration program }\right\} .
$$

The problem $R$ admits the following ILP formulation:

$$
\begin{aligned}
& \min \sum_{e \in E} d(e) \kappa(e) \\
& \text { such that } x(e, w) \leq \kappa(e) \text { for each } e \in E, w \in W, \\
& x_{f}(e, w) \leq \kappa(e) \text { for each } e \in E, w \in W, f \in F, \\
& a_{f} \text { is an integer reassignment for each } f \in F .
\end{aligned}
$$

In addition to the size of the network, the complexity of the above ILP depends on the number of failure scenarios and the size of the associated impact sets. In typical applications, each link failure is considered as a possible failure scenario, and the size of the ILP is large enough to necessitate fast and

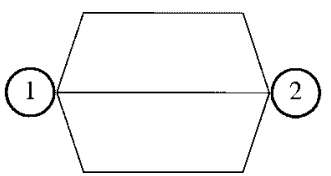

Fig. 4. Sample topology to illustrate the inefficiency of the method of independent designs. It is assumed that $W=8$, and 24 connections are to be established between nodes 1 and 2 .

suboptimal heuristic solution methods. Two such methods are considered next, and their performances are compared in the following section.

\section{A. Method of Independent Designs}

A straightforward heuristic solution for problem $R$ entails decoupling the problem into $|F|$ independent network design problems, one for each failure scenario $f \in F$. In particular, for each failure scenario $f$, one adopts an reconfiguration that would be optimal in the case $F=\{f\}$. Namely, the method of independent designs prescribes the restoration program $\left(a_{f}^{*}: f \in F\right)$, where $a_{f}^{*}$ denotes a solution for the following ILP:

$$
\min \sum_{e \in E} d(e) \kappa(e)
$$

such that $x(e, w) \leq \kappa(e)$ for each $e \in E, w \in W$, $x_{f}(e, w) \leq \kappa(e)$ for each $e \in E, w \in W$,

$a_{f}$ is an integer reassignment for $\{f\}$.

If the size of each impact set is small relative to the total number of connections, then the method of independent designs results in significant reduction in computational complexity. However, since the reassignments $\left(a_{f}^{*}: f \in F\right.$ ) are obtained in an oblivious fashion, this method may lead to restoration programs with high redundant capacity and thus high cost. To illustrate this on an example, consider the topology of Fig. 4, where $F$ consists of the three single-link failures. Assume that the number of wavelengths is 8 , and 24 connections are to be established between the two nodes, so that an optimal primary-network design yields one fiber per link. For definitiveness, assume also that the impact set of a failure is the set of connections on the associated link. The method of independent designs may conceivably result in one redundant fiber on each link, whereas it actually suffices to have one redundant fiber on only two of these links. This observation motivates coordinated heuristic design methods, one of which is the subject of the following subsection.

\section{B. Heuristic Algorithm for Coordinated Designs}

An adaptation of the primary-network design algorithm of Section II-B provides an approximate solution method for the restorable-network design problem. Description of the method relies on a metric defined for each lightpath in the network. Namely, given a restoration scheme $A_{F}$, the metric $M^{*}(p, w)$ of a lightpath $(p, w)$ is defined as

$$
M^{*}(p, w)=\sum_{e \in p} m^{*}(e, w)
$$




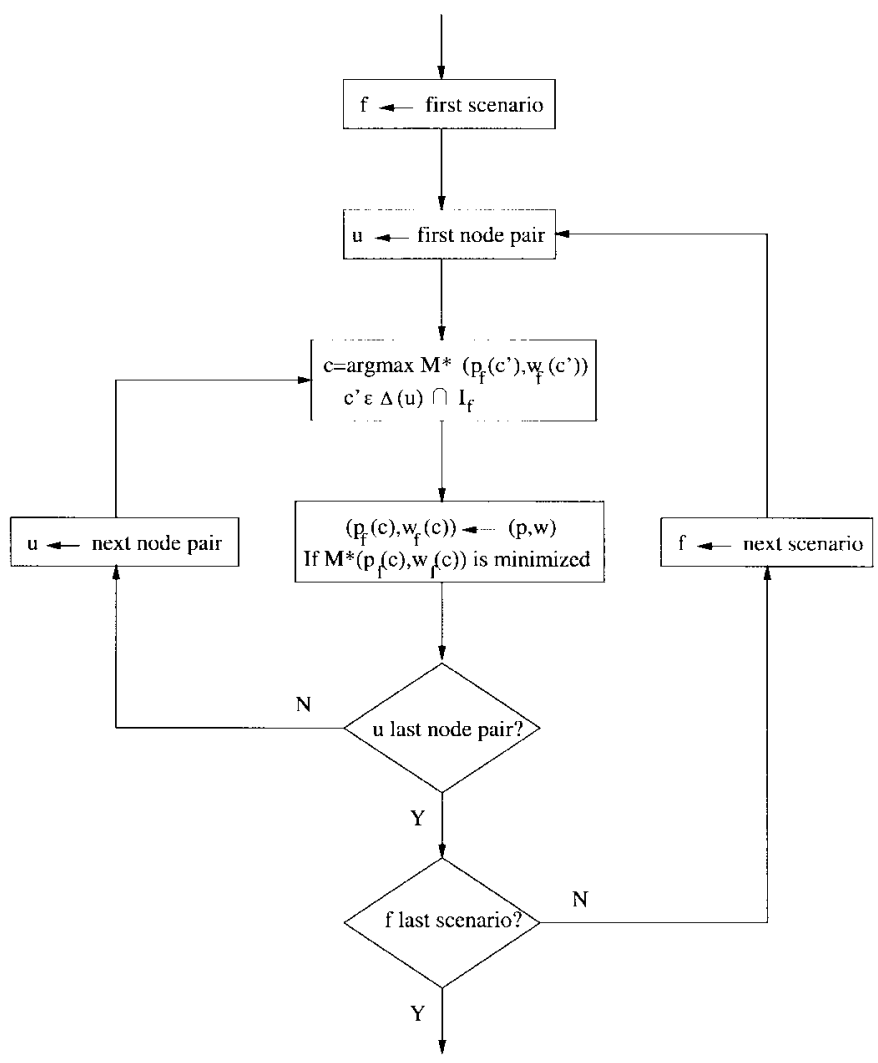

Fig. 5. Flowchart of an iteration of the restorable-network design algorithm.

where

$$
m^{*}(e, w)=\left\{\begin{aligned}
d(e) / n_{e}^{*}\left(A_{F}\right), & \\
& \text { if } x(e, w)^{*}=\max _{w^{\prime} \in W} x^{*}\left(e, w^{\prime}\right) \\
0, & \text { else }
\end{aligned}\right.
$$

and $n_{e}^{*}\left(A_{F}\right)=\left|\left\{\tilde{w} \in W: x^{*}(e, \tilde{w})=\max _{w^{\prime}} x^{*}\left(e, w^{\prime}\right)\right\}\right|$. Note that $n_{e}^{*}\left(A_{F}\right)$ is the number of wavelengths on link $e$ that would become fully loaded under some failure scenario $f \in F$, provided that the restoration program $A_{F}$ is adopted.

We now describe the algorithm. The algorithm maintains one reconfiguration for each failure scenario. The pair $\left(p_{f}(c), w_{f}(c)\right)$ denotes the lightpath assignment of connection $c$ in the current reconfiguration for failure scenario $f$. All reconfigurations are initially set equal to the primary-network configuration. The algorithm then iteratively computes a sequence of restoration programs, until a certain stopping criterion is satisfied. An iteration of the algorithm involves consideration of failure scenarios in a particular order. For each failure scenario $f$, one decision is executed by each node pair; namely, node pair $u$ identifies a connection $c \in \Delta(u) \cap I_{f}$ that maximizes the metric $M^{*}\left(p_{f}(c), w_{f}(c)\right)$, and then reroutes that connection by choosing a restoration path in $P_{f}(c)$ and a wavelength in $W$ so that $M^{*}\left(p_{f}(c), w_{f}(c)\right)$ is minimized under the resulting restoration program. The flowchart of the algorithm is given in Fig. 5. The output of the algorithm is a restoration program that achieves the smallest cost in the course of the iterations. Note that the metric of a lightpath is determined by all assignments in the current restoration program, in turn developments of distinct reconfigurations are coupled with each other.
At any stage of the algorithm the quantity $\max _{x \in W} x^{*}(e, w)$ denotes the capacity requirement of link $e$ under the current restoration program. By definition (4), a lighthop contributes to a lightpath metric only if there is a failure scenario under which the lighthop becomes fully loaded. In this case, the contribution of the lighthop to a lightpath metric is inversely proportional to the number of similar lighthops on the same link. Therefore, qualitatively speaking, by seeking a lightpath with the minimum metric, each rerouting action promotes high wavelength utilization. Note that in the example of Fig. 4, the algorithm finds the optimal solution in one iteration. This algorithm typically performs significantly better than the method of independent designs, as can be observed by the numerical results given in the following section.

The algorithm can be easily extended to account for a richer set of failure types. In particular, lighthop failures can be incorporated by specifying restoration lightpaths instead of restoration paths, and single-fiber failures can be incorporated by applying the algorithm on a new network topology in which each link corresponds to a fiber in the primary network configuration. In the latter case, a single-fiber failure in the original network would be regarded as a link failure in the new network. Note that while both failure types can be crudely handled as link failures, the above modifications yield more efficient designs at the expense of increased computational complexity.

\section{A NUMERICAL STUDY}

This section focuses on a numerical study of the heuristic algorithms introduced in Sections II and III. The heuristic primary-network design algorithm of Section II-B is compared with the optimal solution of the design problem, as well as a commercially available, general purpose heuristic algorithm for solving linear integer problems. The variations introduced in Section II-C are also evaluated. Finally the two restorablenetwork design algorithms of Section III are studied under several restoration schemes.

The numerical results reported in this section are based on simulations that are conducted on the network topology of Fig. 6. This topology is comprised of 32 nodes and 50 links. The weight of each link is taken as the distance of the link, hence the objective of the design problem is to minimize the total fiber-miles in the network. The number of admissible paths is identical for each node pair, and is denoted by $k$. The set of admissible paths is taken as the first $k$ shortest paths.

The optimal cost and the cost obtained by the heuristic primary-network design algorithm of Section II-B are given in Table IV, for several values of $k$. In all cases, the heuristic solutions are obtained within 200 iterations, and the optimal solutions are calculated via the CPLEX software package. ${ }^{3}$ Table IV(a) summarizes our observations in the case when a total of 200 connections are established and the number of wavelengths is 8 : for $k=1$ the optimal solution was obtained in $355(\mathrm{cpu}) \mathrm{s}$ on a Sparc20 workstation. For $k=2$ and $k=3$, the optimal solution could not be obtained within a week;

${ }^{3}$ CPLEX Optimization Inc., NV, USA. 


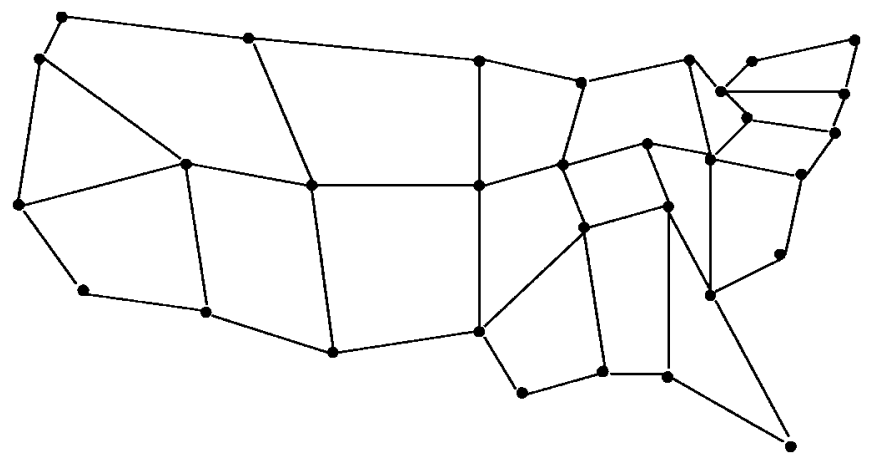

Fig. 6. The network topology considered in the numerical study.

TABLE IV

Costs Obtained by the Optimal and Heuristic Algorithms when (a) 200 CONNECTIONS ARE Established With 8 WAVELENGThs, (b) 200 CONNECTIONS ARE ESTABLISHED WITH 16 WAVELENGHTS, AND (c) 800 CONNECTIONS ARE Established with 32 Wavelengths. The HeuRistic Algorithm Runs Much Faster than the Optimal Algorithm, as Explained in the TeXt

\begin{tabular}{l|c|c|c|c}
\hline \multirow{2}{*}{} & \multicolumn{2}{|c|}{ Optimal } & \multicolumn{2}{c}{ Heuristic } \\
\cline { 2 - 5 } & cost & time & cost & time \\
\hline$k=1$ & 34,970 & $355 \mathrm{sec}$ & 34,970 & $8 \mathrm{sec}$ \\
$k=2$ & $30,160 \geq \geq 29,300$ & $\sim$ hours & 31,240 & $16 \mathrm{sec}$ \\
$k=3$ & $30,160 \geq \geq 27,160$ & $\sim$ day & 31,460 & $24 \mathrm{sec}$ \\
$k=\infty$ & - & - & 30,460 & $420 \mathrm{sec}$ \\
\hline
\end{tabular}

(a)

\begin{tabular}{l|c|c|c|c}
\hline \multirow{2}{*}{} & \multicolumn{2}{|c|}{ Optimal } & \multicolumn{2}{c}{ Heuristic } \\
\cline { 3 - 5 } & cost & time & cost & time \\
\hline$k=1$ & 23,617 & $1400 \mathrm{sec}$ & 23,617 & $19 \mathrm{sec}$ \\
$k=2$ & $20,677 \geq \geq 15,370$ & $\sim$ hours & 20,056 & $39 \mathrm{sec}$ \\
$k=3$ & $20,004 \geq \geq 15,370$ & $\sim$ day & 19,864 & $62 \mathrm{sec}$ \\
$k=\infty$ & - & - & 17,935 & $4760 \mathrm{sec}$ \\
\hline
\end{tabular}

(b)

\begin{tabular}{l|c|c|c|c}
\hline \multirow{2}{*}{} & \multicolumn{2}{|c|}{ Optimal } & \multicolumn{2}{c}{ Heuristic } \\
\cline { 2 - 5 } & cost & time & cost & time \\
\hline$k=1$ & 35,033 & 10 hours & 35,033 & $128 \mathrm{sec}$ \\
$k=2$ & $\geq 22,230$ & - & 30,619 & $270 \mathrm{sec}$ \\
$k=3$ & $\geq 22,230$ & - & 30,801 & $432 \mathrm{sec}$ \\
$k=\infty$ & - & - & 30,659 & $\sim$ day \\
\hline
\end{tabular}

(c)

therefore, only upper and lower bounds, provided by the same package, are provided for these cases. For $k=2$, an integer assignment with cost 30160 was obtained in a few hours, and for $k=3$, an integer assignment with cost 44335 was obtained in a day. Since the integer assignment obtained for the case $k=2$ is also admissible for the case $k=3$, the value 30160 is registered as an upper bound for the latter case. The corresponding heuristic solutions were obtained in roughly $k \times 8$ (cpu) s. Similar observations were made about establishing 200 connections with 16 wavelengths, as illustrated in Table IV(b). When a larger problem with 800 connections and 32 wavelengths was considered, the CPLEX algorithm computed the optimal assignment in about $10 \mathrm{~h}$ for $k=1$. However, for larger values of $k$, it failed to generate any integer assignments within a week. In contrast, the heuristic algorithm generated solutions in roughly $k \times 130(\mathrm{cpu}) \mathrm{s}$, as indicated in Table IV(c). The lower bounds of Table IV(b) and (c) are obtained via the LP relaxation of the network design problem.

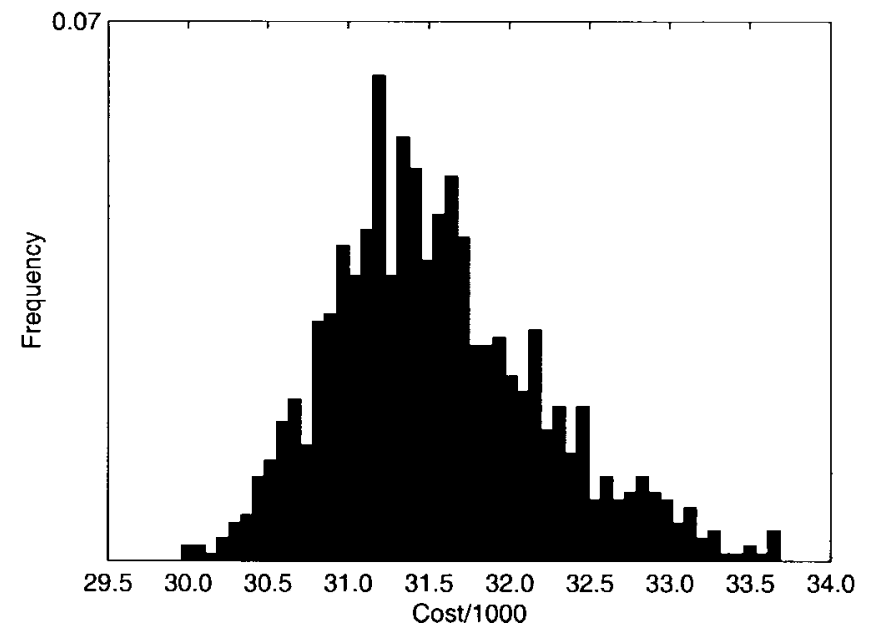

Fig. 7. The distribution of the final cost obtained by the algorithm, over 1000 randomly selected initial configurations.

The solution obtained by the algorithm seems to have weak dependence on the initial configuration. Fig. 7 sketches the distribution of the cost corresponding to 1000 randomly chosen initial configurations, in the setting of Table IV(a) (200 connections with eight wavelengths) with $k=3$. The best and the worst initial configurations resulted in costs of 29959 and 33687 , respectively, yielding roughly $12.4 \%$ sensitivity on the initial configuration. The average cost was 31552 . In the experiments reported in Table IV, initial configurations are built up from an empty network by sequentially routing connections on admissible lightpaths with smallest metric in the current partially configured network. In the setting of Table IV(a), the resulting cost was within $5.0 \%$ of the best cost over the 1000 initial configurations. It is also remarkable that the sequential build-up method consistently resulted in better initial configurations when longer connections are routed first.

The algorithm prescribes rerouting one connection per node pair per iteration, rather than rerouting all connections of a node pair and then proceeding with the next node pair. Intuitively, this serves the purpose of "shuffling" the rerouting actions taken by the node pairs so as to provide better coordination among these actions. Numerical experiments suggest that somewhat better performance may be expected by further coordinating node pairs via rerouting connections in the order of increasing metric. This approach, of course, entails considerably higher computational complexity.

The value of the optimal solution is minimal in the case $k=\infty$, when any path connecting a given pair of nodes is admissible for that pair. Note that the problem can then be formulated as a multicommodity flow problem with integral constraints. While this case entails a high complexity for the ILP formulation, it can be handled efficiently by a simple variation of the heuristic algorithm. In particular, each selected connection is first removed from the network, then an admissible lightpath that has the minimum metric is found via Dijkstra's shortest path algorithm, and finally the connection is rerouted on that lightpath. The last rows in Table IV(a)-(c) give the values obtained by this approach.

The metric of a lightpath is comprised of contributions $m(e, w)$ from lighthops $(e, w)$ that are maximally loaded in the sense that $x(e, w)=\max _{w^{\prime}} x\left(e, w^{\prime}\right)$. Furthermore, 

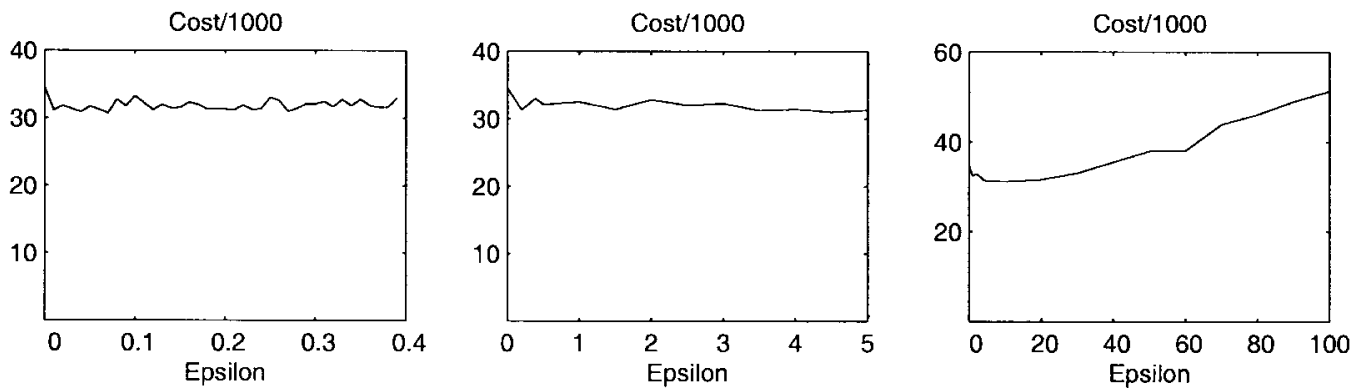

Fig. 8. Variation of the cost obtained by the algorithm with respect to $\epsilon$.

if lighthop $(e, w)$ is maximally loaded, then $m(e, w)$ is inversely proportional to the number of similar lighthops on link $e$. Therefore, by rerouting connections on lightpaths with minimal metrics, a rerouting action effectively seeks to equalize the loads $x(e, w)$ for each link $e$, and thereby promotes high wavelength utilization. High wavelength utilization, however, may not imply low network cost, since it may possibly be achieved at the expense of routing connections on unnecessarily long lightpaths. Such a situation is likely to arise if the number of admissible paths per node pair is large so that some paths are considerably longer than others. To avoid undesirable configurations in which high wavelength utilization is obtained at the expense of high network cost, it is reasonable to modify the metric $M$ by setting

$m(e, w)= \begin{cases}d(e) / n_{e}(a), & \text { if } x(e, w)=\max _{w^{\prime} \in W} x\left(e, w^{\prime}\right) \\ \epsilon, & \text { else }\end{cases}$ for a small positive $\epsilon$, or to replace $M$ by $M^{\alpha}$ for a suitably large value of $\alpha$. Fig. 8 sketches the variation of the cost when $\epsilon$ is varied in several scales. Here, the setting of Table IV(a) is considered with $k=\infty$. When $\epsilon=0$, the best configuration obtained by the algorithm in 200 iterations has cost 34526 . Fig. 8 suggests that even very small positive values of $\epsilon$ result in penalizing longer paths, and provide an immediate $10 \%$ cost improvement. For large values of $\epsilon$, however, all lightpath metrics are altered significantly, in turn the cost deteriorates. When $\epsilon$ varies within moderate limits, the cost displays small and rather unpredictable variations, very much like it does in the case of different initial configurations. This appears to be due to the large number of fixed points of the algorithm. In turn, we somewhat arbitrarily chose a small $\epsilon$ or a large $\alpha$ in our experiments.

We now consider the merits of the primary-network design algorithm relative to a general purpose heuristic solution method that is provided in the CPLEX package. The CPLEX heuristic is based on pre-assigning integer values to a number of variables, using a rounding argument on a solution of the LP relaxation of the problem. A reduced ILP of a manageable size is thereby obtained. The major drawback of this approach is that it may not lead to integer assignments. This issue arises consistently in large problems. Here, we concentrate on a range of the total number of connections for which the CPLEX heuristic produced integer assignments. The resulting costs and time complexities of the two heuristics are plotted in Fig. 9. In the simulations $k=2$, the number of wavelengths is 8 , and the data are obtained by averaging over ten different traffic demands.

Fig. 10 plots the network cost obtained by the primarynetwork design algorithm and its five variations introduced in
Cost

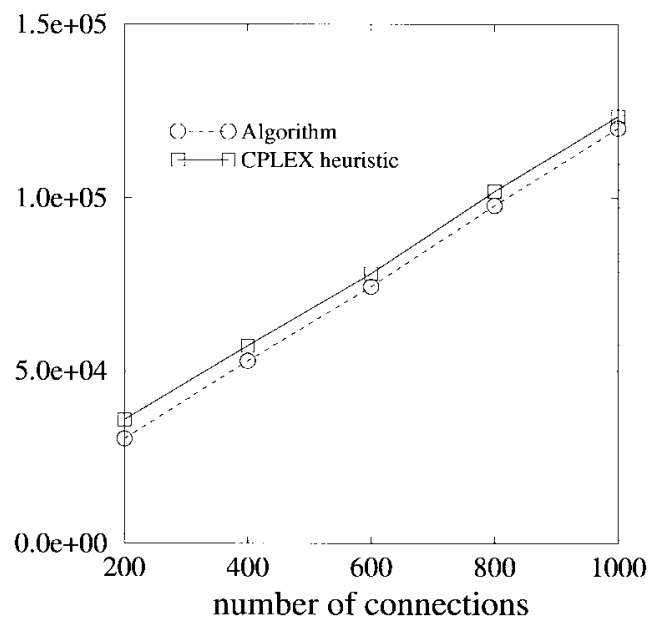

(a)

Cpu seconds

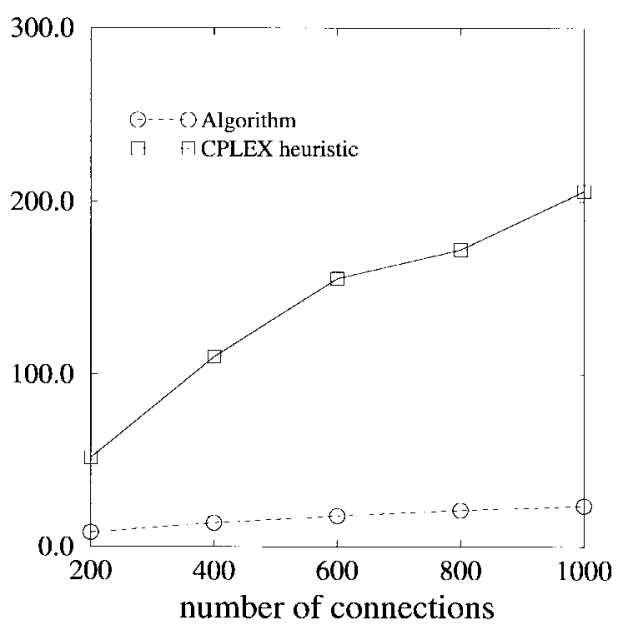

(b)

Fig. 9. A comparison of the proposed algorithm and the CPLEX heuristic for primary-network design.

Section II-C. Here $k=2$, and 200 connections are established with 8 wavelengths. All variations except $m N P$ provided relatively better results when the metrics are evaluated before assignment, thus the corresponding values are reported in the figure. Algorithm $m N S$ performed quite well, whereas the remaining variations performed rather poorly. In all cases, the average wavelength utilization, i.e., the ratio of the number of utilized lighthops to the number of available lighthops, 


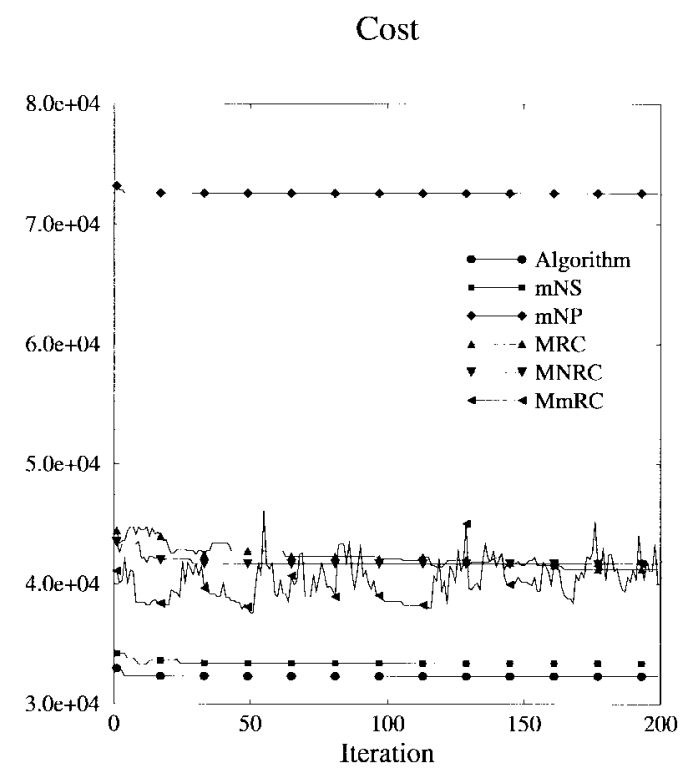

Fig. 10. Network cost obtained by various versions of the primary-network design algorithm.

was around $80 \%$. These observations agree with the intuitive discussion of Section II-C.

In the rest of this section we focus on the design of restorable networks. Three different restoration schemes are considered for these networks: in the full-reconfiguration scheme, all connections are allowed to be reconfigured upon a failure, whereas in the path-based and link-based restoration schemes, a connection can be reconfigured only if it uses failed components in the primary-network configuration. In full-reconfiguration and path-based schemes, a reconfigured connection is assigned to one of the $k$ shortest paths in the faulty network. In the link-based scheme, such a connection retains the functional segment of its original lightpath and bypasses each failed network component by using one of the $k$ appropriate shortest paths.

In practice, routing a connection requires communication and configuration of the switches along the lightpath. Hence there is an inevitable delay, generally referred to as the restoration time, to program a new reconfiguration into the network. This delay depends mainly on the selection of restoration paths. Typically, the restoration time of full reconfiguration is no less than that of path-based reconfiguration, since the latter fewer connections are involved in the restoration process, and the restoration time of path-based reconfiguration is no less than that of link-based reconfiguration, since the latter few switches are reconfigured per restored connection. We shall not address restoration time in detail here; instead, we shall concentrate on the capacity requirement under the three restoration schemes.

We now provide a numerical comparison of the two restorable-network design algorithms introduced in Section III. We take $k=2$, and consider the two problems of provisioning 200 connections with 8 wavelengths, and provisioning 800 connections with 32 wavelengths. The set of failure scenarios $F$ is taken to be the set of all single-link failures, and the three restoration schemes of the previous paragraph are considered. The heuristic algorithm of Section II-B is employed to obtain
TABLE V

Total Redundant CAPaCity (In Fiber-Miles), NoRmalized by Dividing With the Total Working Capacity (in Fiber-Miles), Under the Considered Restoration Schemes and Design Algorithms. (a) 200 ConNeCtions with 8 WAVELENGTHS AND (b) 800 CONNECTIONS WITH 32 WAVELENGTHS

\begin{tabular}{l|c|c}
\hline & Independent & Coordinated \\
\hline Full-reconfiguration & 0.92 & 0.70 \\
Path-based & 1.01 & 0.87 \\
Link-based & 1.43 & 1.23 \\
\hline \multicolumn{3}{|c}{} \\
\hline \multicolumn{3}{|c}{} \\
\hline Full-reconfiguration & Independent & Coordinated \\
Path-based & 0.84 & 0.66 \\
Link-based & 0.91 & 0.74 \\
\hline
\end{tabular}

the primary-network configuration, as well as each individual reconfiguration in the method of independent designs. The ratio of the redundant fiber-miles to the working fiber-miles obtained in each case is given in Table V. Note that in each of the settings (a) and (b), the primary-network design, and hence the working fiber miles, is identical for all schemes and algorithms. The full reconfiguration and link-based schemes required, respectively, the least and most fiber miles, and the coordinated heuristic resulted in roughly a $20 \%$ savings in spare capacity in each restoration scheme.

\section{CONCLUSION}

This paper concerned two heuristic methods for routing and wavelength assignment of static connections in WDM optical networks. The first method is an iterative algorithm which is suitable for networks that do not account for failures. The algorithm is based on greedy decisions by the connections to decrease a certain metric whose minimum value corresponds to an optimal assignment. The computational complexity per iteration of the algorithm is low, and it is shown numerically on a mesh-like topology that the algorithm generates efficient solutions in a reasonable number of iterations. The second method concerns the design of fault-tolerant networks, and is obtained by an adaptation of the first method. The method entails coordinated planning of several failure scenarios, and a numerical study shows that it provides more efficient designs than those obtained by considering the failures independently.

\section{APPENDIX}

Proof of Proposition 2.1: Let $a_{1}, a_{2}$ be assignments with respective loads $x_{1}, x_{2}$, and let $0<\beta<1$. Then

$$
\begin{aligned}
& J^{\alpha}\left(\beta a_{1}+(1-\beta) a_{2}\right) \\
& =\alpha^{-1} \sum_{e \in E} \log \sum_{w \in W} \\
& \cdot \exp \left(\beta \alpha x_{1}(e, w)+(1-\beta) \alpha x_{2}(e, w)\right) \\
& \leq \alpha^{-1} \sum_{e \in E} \log \left\{\left(\sum_{w \in W} \exp \left(\alpha x_{1}(e, w)\right)\right)^{\beta}\right. \\
& \left.\cdot\left(\sum_{w \in W} \exp \left(\alpha x_{2}(e, w)\right)\right)^{(1-\beta)}\right\} \\
& =\beta J^{\alpha}\left(a_{1}\right)+(1-\beta) J^{\alpha}\left(a_{2}\right)
\end{aligned}
$$


where the second step follows by Hölder's inequality. In particular, $J^{\alpha}$ is convex, and $P^{\alpha}$ is a convex optimization problem on a compact, convex set; thus, it has a solution. To complete the proof, it suffices to show that condition (2) is sufficient for optimality in $P^{\alpha}$. Toward this end, let $a$ denote an assignment that satisfies (2), and assume that $a$ is not optimal. Then by the convexity of $J^{\alpha}$, there exists a perturbation vector $h=(h(p, w): p \in P(u), u \in U, w \in W)$ such that

$$
\begin{aligned}
& \sum_{p \in P(u)} \sum_{w \in W} h(p, w)<0 \text { only if } a(p, w)>0 \\
& \lim _{\epsilon \backslash 0} \frac{J^{\alpha}(a+\epsilon h)-J^{\alpha}(a)}{\epsilon} \\
& =\sum_{u \in U} \sum_{p \in P(u)} \sum_{w \in W} h(p, w) M^{\alpha}(p, w)<0 .
\end{aligned}
$$

Let $u \in U$ be arbitrary, and define $M_{+}^{\alpha}(u)=$ $\min \left\{M^{\alpha}(p, w): p \in P(u), w \in W, h(p, w)>0\right\}$ and $M_{-}^{\alpha}(u)=\max \left\{M^{\alpha}(p, w): p \in P(u), w \in W, h(p, w)<\right.$ $0\}$. Condition (5) and the hypothesis that $a$ satisfies condition (2) imply that $M_{+}^{\alpha}(u) \geq M_{-}^{\alpha}(u)$, and in turn

$$
\begin{aligned}
& \sum_{p \in P(u)} \sum_{w \in W} h(p, w) M^{\alpha}(p, w) \\
& =\sum_{p \in P(u)} \sum_{w \in W} h(p, w) I\{h(p, w)>0\} M^{\alpha}(p, w) \\
& \quad+\sum_{p \in P(u)} \sum_{w \in W} h(p, w) I\{h(p, w)<0\} M^{\alpha}(p, w) \\
& \geq \sum_{p \in P(u)} \sum_{w \in W} h(p, w) I\{h(p, w)>0\} M_{+}^{\alpha}(u) \\
& \quad+\sum_{p \in P(u)} \sum_{w \in W} h(p, w) I\{h(p, w)<0\} M_{-}^{\alpha}(u) \\
& =\sum_{p \in P(u)} \sum_{w \in W} h(p, w) I\{h(p, w)>0\} \\
& \quad \cdot\left(M_{+}^{\alpha}(u)-M_{-}^{\alpha}(u)\right) \\
& \geq 0
\end{aligned}
$$

where the third step is followed by condition (6). This contradicts with (7), and thus, with the existence of $h$. Therefore, condition (2) is sufficient for optimality, and the proof is complete.

\section{REFERENCES}

[1] S. B. Alexander, R. S. Bondurant, D. Byrone, V. W. S. Chan, S. G. Finn, R. Gallager, B. S. Glance, H. A. Haus, P. Humblet, R. Jain, I. P. Kaminow, M. Karol, R. S. Kennedy, A. Kirby, H. Q. Le, A. A. M. Saleh, B. A. Schofield, J. H. Shapiro, N. K. Shankaranarayanan, R. E. Thomas, R. C. Williamson, and R. W. Wilson, "A precompetetive consortium on wide-band all-optical networks," J. Lightwave Technol., vol. 11, pp. 714-735, May 1993.

[2] G. K. Chang, G. Ellinas, J. K. Gamelin, M. Z. Iqbal, and C. A. Brackett, "Multiwavelength reconfigurable WDM/ATM/SONET network testbed," J. Lightwave Technol., vol. 14, pp. 1320-1340, June 1996.

[3] R. E. Wagner, R. C. Alferness, A. A. M. Saleh, and M. S. Goodman, "MONET: Multiwavelength optical networking," J. Lightwave Technol., vol. 14, pp. 1349-1355, June 1996.
[4] I. Chlamtac, A. Ganz, and G. Karmi, "Lightpath communications: An approach to high bandwidth optical WAN's," IEEE Trans. Commun., vol. 40, pp. 1171-1182, July 1992.

[5] N. Nagatsu, Y. Hamazumi, and K. Sato, "Number of wavelengths required for constructing large-scale optical path networks," Electron. Commun. Jpn., pt. 1, vol. 78, no. 9, pp. 1-11, Sept. 1995.

[6] D. Banerjee and B. Mukherjee, "A practical approach for routing and wavelength assignment in large wavelength routed optical networks," IEEE J. Select Areas Commun., vol. 14, pp. 903-908, June 1996.

[7] N. Nagatsu and K. Sato, "Optical path accommodation design enabling cross-connect system scale evaluation," IEICE Trans. Commun., vol. E78-B, no. 9, pp. 1339-1343, Sept. 1995.

[8] G. Jeong and E. Ayanoglu, "Comparison of wavelength-interchanging and wavelength-selective cross-connects in multiwavelength all-optical networks," in Proc. IEEE INFOCOM'96, pp. 156-163.

[9] R. Ramaswami and K. N. Sivarajan, "Routing and wavelength assignment in all-optical networks," IEEE/ACM Trans. Networking, vol. 3, pp. 489-500, Oct. 1995.

[10] R. Dighe, Q. Ren, and B. Sengupta, "A link based alternative routing scheme for network restoration under failure," in Proc. GLOBECOM'95, Nov. 1995, pp. 2118-2123.

[11] W. D. Grover, T. D. Bilodeau, and B. D. Vanables, "Near optimal spare capacity planning in a mesh restorable network," in Proc. GLOBECOM'91, pp. 2007-2011.

[12] B. D. Venables, W. D. Grover, and M. H. MacGregor, "Two strategies for spare capacity placement in mesh restorable networks," in Proc. ICC'93, pp. 267-271.

[13] N. Nagatsu, S. Okamoto, and K. Sato, "Optical path cross-connect scale evaluation using path accommodation design for restricted wavelength multiplexing," IEEE J. Select. Areas Commun., vol. 14, pp. 893-901, June 1996.

[14] K. Sato and N. Nagatsu, "Failure restoration in photonic transport networks using optical paths," in Proc. OFC'96, pp. 215-216.

[15] L. Wuttisittikulkij and M. J. O'Mahony, "Use of spare wavelength for traffic restoration in multi-wavelength transport network," in Proc. ICC'95, pp. 1778-1782.

[16] D. Bertsekas and R. Gallager, Data Networks. Englewood Cliffs, NJ: Prentice-Hall, 1992.

[17] E. Karasan and E. Ayanoglu, "Effects of wavelength routing and selection algorithms on wavelength conversion gain in WDM optical networks," in Proc. IEEE GLOBECOM'96, London, England, vol. 1, pp. 299-305, Nov. 1996.

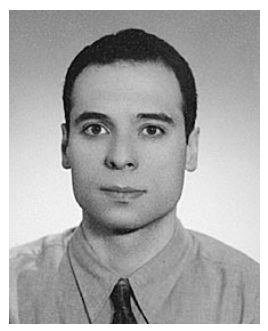

Murat Alanyali (S'89-M'97) received the B.Sc degree from Middle East Technical University, Ankara, Turkey, the M.Sc. degree from Bilkent University, Ankara, Turkey, and the Ph.D. degree from the University of Illinois at Urbana-Champaign, all in electrical engineering.

From 1996 to 1997, he was with the Broadband Systems Research Department, Bell Laboratories, Lucent Technologies. He is currently an Assistant Professor of Electrical and Electronics Engineering at Bilkent University. His research focuses on the design and management of high-speed, optical networks.

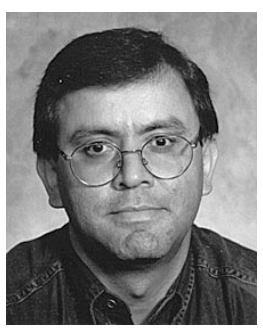

Ender Ayanoglu (S'82-M'85-SM'90-F'98) received the B.S. degree from Middle East Technica University, Ankara, Turkey, in 1980, and the M.S. and Ph.D. degrees from Stanford University, Stanford, CA, in 1982 and 1986, respectively, all in electrical engineering.

From October 1986 to April 1999, he was a Member of Technical Staff at Bell Laboratories, Lucent Technologies. He is currently a Technical Leader at Cisco Systems, San Jose, CA.

Dr. Ayanoglu is the Chair of the IEEE Communication Society Communication Theory Technical Committee and the Editor for Communication Theory and Coding Applications of the IEEE TRANSACtions on Communications. He is the co-recipient of the 1995 IEEE Communications Society Stephen O. Rice Prize Paper Award and the 1997 IEEE Communications Society Best Tutorial Paper Award. 